\title{
Asymptomatic Secondary Aortoduodenal Fistula Incidentally Diagnosed During a Health Check-up: A Case Report
}

\author{
Sang Ki Park ${ }^{1}$, In Chul Min², Sung Hoon Lee ${ }^{1}$ Jae Ik Lee ${ }^{1}$, Ji Min Kim³, Kyong Hye Joung ${ }^{3}$, Bon Jeong Ku ${ }^{3}$ \\ Departments of ${ }^{1}$ Internal Medicine and ${ }^{2}$ General Surgery, City Clinic, Daejeon, ${ }^{3}$ Department of Internal Medicine, Chungnam National \\ University School of Medicine, Daejeon, Korea
}

Corresponding Author: Bon Jeong $\mathrm{Ku}, \mathrm{MD}, \mathrm{PhD}$ Department of Internal Medicine, Chungnam National University School of Medicine, 282 Munhwa-ro, Jung-gu, Daejeon 35015, Korea

Tel: +82-42-280-7149

Fax: +82-42-280-6990

E-mail: bonjeong@cnu.ac.kr

Received: January 23, 2018 Revised: January 23, 2018 Accepted: February 20, 2018

\begin{abstract}
Secondary aortoenteric fistulas (AEFs) are an uncommon but fatal complication of abdominal aortic reconstructive surgery. Aortoduodenal fistulas (ADFs) are the most frequent secondary AEFs (80\%). The incidence of secondary AEFs is increasing because of the increasing number of individuals undergoing aortic aneurysm repairs with prosthetics. In most cases of secondary AEFs, surgical repair is performed with the patient in a poor condition, owing to late diagnosis. Thus, an early diagnosis is crucial. We report a case of an asymptomatic secondary ADF that was incidentally diagnosed using esophagogastroduodenoscopy (EGD) and computed tomography during a health check-up and was successfully repaired, with the patient in a stable condition even after the development of life-threatening symptoms. Based on our case, we suggest that noticing unexplained leukocytosis in regular hematological examination and performing surveillance EGD in an asymptomatic individual who has undergone abdominal aortic reconstructive surgery may aid in the early diagnosis of secondary AEFs before fatal complications manifest.
\end{abstract}

Key Words: Aorta, Duodenum, Fistula, Asymptomatic Disease

\section{INTRODUCTION}

Aortoenteric fistulas (AEFs) are defined as abnormal communications between the intestine and the aorta. They are classified as either primary or secondary AEFs. ${ }^{1)}$ Aortoduodenal fistulas (ADFs) are the most frequent type of secondary AEFs, with an incidence of $80 \%,{ }^{2)}$ and the most commonly involved sites are the third and fourth portions of the duodenum $(60 \%)^{2)}$ AEFs occur in $0.3 \%-2 \%$ of patients undergoing abdominal aortic reconstructive surgery. ${ }^{3)}$ This complication could occur within 2 days to more than 14 years after abdominal aortic reconstructive surgery. ${ }^{4)}$ The clinical features of secondary AEFs are gastrointestinal bleeding (80\%), abdominal pulsatile mass (56\%), sepsis (44\%), and abdominal pain (30\%). ${ }^{5)}$

Owing to the high surgical mortality with the late diagnosis of AEFs and the $100 \%$ mortality rate without surgical repair, better diagnostic tools and other therapeutic modalities should be further explored. Currently, however, the only unique way to reduce mortality and save the life of a patient with secondary AEFs is to ensure an early diagnosis before life-threat- ening symptoms develop, and to perform early surgical repair.

In this case, AEF was incidentally diagnosed during a health check-up using esophagogastroduodenoscopy (EGD) and computed tomography $(\mathrm{CT})$, and was successfully repaired.

\section{CASE REPORT}

A 72-year-old man first visited our clinic for a health checkup on January 20, 2014. He was asymptomatic at that time and was taking antihypertensive and antiplatelet drugs, and statins.

Physical examination did not reveal any unusual findings except for a median linear scar on the abdomen of the patient owing to a previous abdominal surgery. He had undergone aorto-bifemoral bypass 75 months ago because of atherosclerosis obliterans. His vital signs were as follows: pulse rate, 60/min; respiratory rate, 17/min; body temperature, $36.8^{\circ} \mathrm{C}$; and blood pressure, $125 / 69 \mathrm{mmHg}$. Complete hematological examination revealed unremarkable results, except for mild leukocytosis $(14,000 / \mu \mathrm{L})$ and mild normocytic normochromic anemia (hemoglobin, $11.4 \mathrm{~g} / \mathrm{dL}$ ).

Routine EGD showed a pulsating aortic prosthesis perfora- 
ting the third portion of the duodenum, without any signs of bleeding or obstruction (Fig. 1A). As the condition of the patient was stable, enhanced CT was performed to evaluate the location and condition of the fistula. CT images showed the prosthesis penetrating the third portion of the duodenum, loss of the normal fat distribution pattern between the aortic prosthesis and the duodenum, and ectopic gas adjacent to the aortic prosthesis. However, it did not show leakage of aortic contrast media into the eroded duodenal lumen (Fig. 2).

Considering the above findings, the patient was diagnosed with secondary ADF (type 2, paraprosthetic enteric fistula) and promptly transferred to a tertiary hospital for treatment. He underwent laparotomy to remove the prosthetic implant, followed by in situ replacement with a new implant, and duodenal primary repair.

Mild inflammation of the tissues surrounding the prosthetic implant was observed, with no pus. During hospitalization, the patient had no specific adverse events and was discharged 4 weeks after surgical repair.
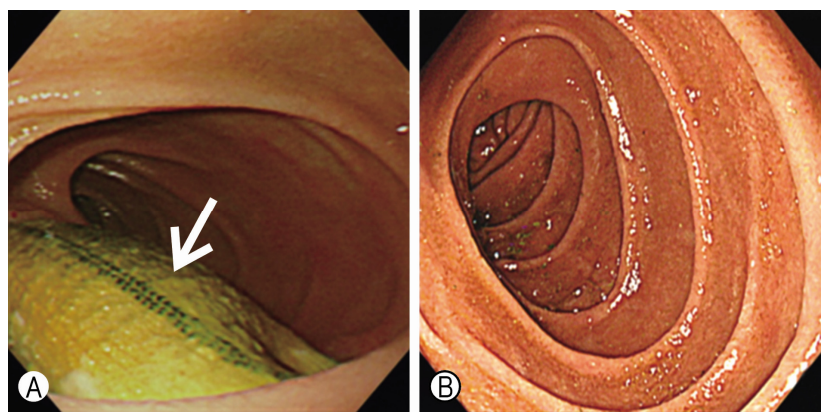

Fig. 1. (A) Preoperative endoscopic image shows prosthesis perforating the third portion of duodenum without bleeding and obstruction sign (arrow). (B) Postoperative endoscopic image does not show the aortic prosthesis that was previously observed in the third portion of the duodenum.
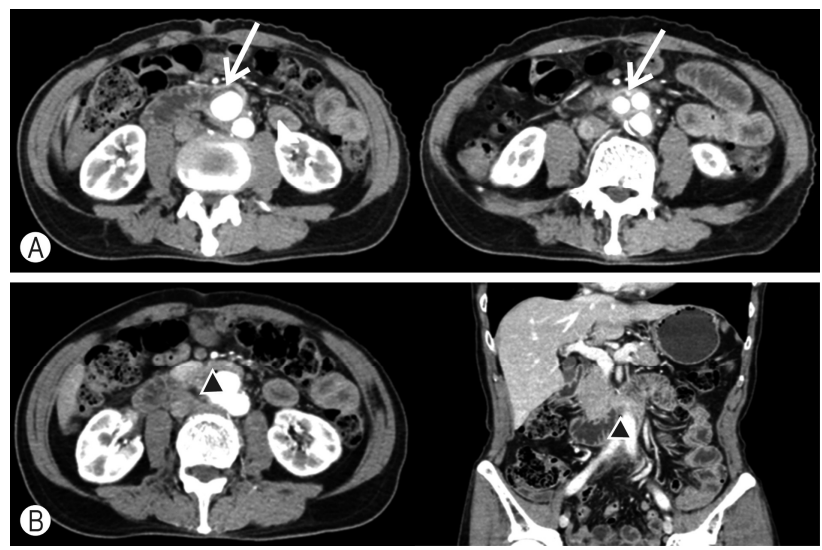

Fig. 2. Computed tomography images show the prosthesis penetrating the third portion of the duodenum (A, arrow) and ectopic gas adjacent to the aortic prosthesis (B, arrowhead).
On March 8, 2016, 2 years after surgery, the patient revisited our clinic for a routine health check-up. He did not have any remarkable symptoms during the second visit as well. Physical examination showed no unusual findings. Complete hematological examination did not show leukocytosis or anemia (white blood cell, 7,800/uL; hemoglobin, $13.2 \mathrm{~g} / \mathrm{dL}$ ). Routine EGD showed that the aortic prosthesis previously observed in the third position of the duodenum had disappeared (Fig. 1B).

Written informed consent has been obtained from the patient described in the case report.

\section{DISCUSSION}

Secondary AEF is defined as a fistula formed between the intestine and the prosthetic implants after abdominal aortic reconstructive surgery. Secondary AEFs occur in a minority of individuals who undergo aortic aneurysm repairs with prosthetic implants. ${ }^{3)}$ Although the duodenum is the most commonly involved site of secondary AEFs $(80 \%){ }_{1}{ }^{2)}$ fistula formations in other organs, such as colon, rectum, and small intestine, have also been reported. ${ }^{3,6)}$ In general, secondary AEFs are classified as types 1 and 2. Type 1 AEF, also called graft enteric fistula or true $A E F$, refers to a fistula occurring between the intestine and the proximal aortic suture line, with or without a pseudoaneurysm. This type of fistula is the most common. Type 2, also called paraprosthetic enteric fistula, refers to an absent fistula between the intestine and the aortic implant. It accounts for $15 \%-20 \%$ of secondary AEFs. ${ }^{6)}$ The main clinical feature of type 2 fistulas is sepsis $(57 \%){ }^{7)}$ In addition to sepsis, gastrointestinal bleeding (30\%), abdominal pain $(20 \%)$, septic arthritis, multicentric osteomyelitis, hypertrophic osteoarthropathy, and septic emboli in the lower extremities have been reported. ${ }^{7,8)}$ The precise pathogenesis of secondary AEF is still unknown; however, the mechanical pressure of the aortic implant on the intestine, septic procedures, or the lack of interposed retroperitoneal tissue between the intestine and the aortic implant are proposed as causative mechanisms. ${ }^{3)}$

As the mortality rate of patients with secondary AEF who do not undergo surgical repair is $100 \%$, early diagnosis and surgical repair are important. Although no accurate diagnostic method for detecting secondary AEF has yet been established, CT and EGD are the most commonly used methods for diagnosis, with the sensitivity of CT being $45 \% .{ }^{5}$ ) Positive CT findings of secondary AEFs include loss of the normal fat distribution pattern between the aortic implants and the intestine, ectopic gas within or adjacent to the aortic implant, leakage of intestinal contrast media into the paraprosthetic space, and leakage of aortic contrast media into the penetrated intestinal lumen. ${ }^{9)}$ The sensitivity of EGD is $24 \%$ for the diagnosis of secondary AEFs. ${ }^{5}$ EGD has been reported 
as a useful diagnostic tool for many stable individuals with secondary AEFs. ${ }^{410)}$ Endoscopic findings of secondary AEF include fistular openings, active arterial bleeding, and prosthesis in the intestinal wall or crossing the intestinal lumen. ${ }^{2)}$ Using a combination of CT and EGD can improve the diagnostic accuracy. ${ }^{2)}$

Most patients with secondary AEFs are diagnosed only after the occurrence of fatal symptoms. However, in our case, secondary ADF (type 2, paraprosthetic enteric fistula) was diagnosed by EGD and CT during routine health check-up at 76 months following aortic reconstructive surgery.

Upon the patient's first visit to our clinic, hematological examination showed mild leukocytosis and mild normocytic normochromic anemia; however, the patient did not exhibit any remarkable clinical symptoms of secondary ADF. Hematological examination performed two years after surgery did not show leukocytosis or anemia. Considering the surgical findings, the preoperative leukocytosis was thought to be caused by inflammation of the tissues surrounding the aortic prosthesis. Anemia observed prior to surgery was not microcytic, but hypochromic anemia was observed owing to the chronic blood loss by secondary AEF. Thus, we believe that this anemia could be attributed to the chronic inflammatory state of the tissues surrounding the aortic prosthesis.

Based on these findings, we believe that the efforts to detect unexplained leukocytosis or microcytic hypochromic anemia through periodic hematological examination and EGD in an asymptomatic individual who underwent abdominal aortic reconstructive surgery are useful for early diagnosis of secondary AEF before fatal symptoms occur.

Conflicts of Interest Disclosures: The researchers claim no conflicts of interest.

\section{REFERENCES}

1. Kim JY, Kim YW, Kim CJ, Lim HI, Kim DI, Huh S. Successful surgical treatment of aortoenteric fistula. J Korean Med Sci 2007; 22:846-50.

2. Grassia R, Staiano T, liritano E, Bianchi G, Dizioli P, Baratta $\mathrm{V}$, et al. Gastrointestinal hemorrhage caused by secondary aortoduodenal fistula: a case report. Eur Rev Med Pharmacol Sci 2009;13:147-50.

3. Geraci G, Pisello F, Li Volsi F, Facella T, Platia L, Modica G, et al. Secondary aortoduodenal fistula. World J Gastroenterol 2008;14:484-6.

4. Galloro G, De Palma GD, Siciliano S, Amato B, Catanzano C. Secondary aortoduodenal fistula. Rare endoscopic finding in the course of digestive hemorrhage. Hepatogastroenterology 2000; 47:1585-7.

5. Iwaki T, Miyatani H, Yoshida Y, Okochi T, Tanaka O, Adachi $\mathrm{H}$. Secondary aortoduodenal fistula without gastrointestinal bleeding directly detected by CT and endoscopy. Radiol Case Rep 2015;7:774.

6. Luo CY, Lai CH, Wen JS, Lin BW. Secondary aortocolic fistula: case report and review of the literature. Ann Vasc Surg 2010;24: 256.e5-12.

7. Dachs RJ, Berman J. Aortoenteric fistula. Am Fam Physician 1992;45:2610-6.

8. Chang MW, Chan YL, Hsieh HC, Chang SS. Secondary aortoduodenal fistula. Chang Gung Med J 2002;25:626-30.

9. Vu QD, Menias CO, Bhalla S, Peterson C, Wang LL, Balfe DM. Aortoenteric fistulas: CT features and potential mimics. Radiographics 2009;29:197-209.

10. Armstrong PA, Back MR, Wilson JS, Shames ML, Johnson BL, Bandyk DF. Improved outcomes in the recent management of secondary aortoenteric fistula. J Vasc Surg 2005;42:660-6. 